\title{
Aesthetic refining with autologous fat graft post breast reconstruction
}

\begin{abstract}
The objective of this study was to verify the aesthetic efficacy of autologous fat graft in the breast reconstruction after refinement, through the presentation of a clinical case report. The methodology was ADOPTED a review of the literature Followed by the clinical case report, where an example of esthetic rehabilitation with autologous fat grafting is addressed in practice. Based on the literature reports and the description of this clinical case, it was concluded que fat grafting provides the improvement of quality skin and aesthetics in general. Although the Desired volume is not reached in the first session, satisfactory results can be obtained from the application of several sessions, adding excellent aesthetic result and improvement of the quality of life for the patients who have undergone this devastating disease nowadays.
\end{abstract}

Keywords: breast reconstruction, mastectomy, fat grafting, autologous fat graft in breast
Volume 8 Issue 2 - 2020

\author{
Laurinda Castellani,' Roberto José da Silva \\ Vieira, ${ }^{2}$ Marcio Paneghini ${ }^{3}$ \\ 'Plastic surgeon Sociedade Brasileira de Plastica Surgery, Brazil \\ 2Professor of Oncoplastia II and Head of the Department of \\ Gynecology of IFF-Fiocruz, Brazil \\ ${ }^{3}$ Head of the Cancer Itapeva, Brazil
}

Correspondence: Laurinda Castellani, Plastic surgeon

Sociedade Brasileira de Plastica Surgery, Brazil,

Email Icastellani@hotmail.com

Received: January 28, 2020 | Published: June 24, 2020

\section{Introduction}

The breast is one of the most important organs in the female identity and sexuality of women. The surgical cancer treatment often leads to ugly situations, causing social and sexual emotional disorders for women. Breast cancer is the most common type among women in the world and in Brazil, accounting for about $28 \%$ of new cases each year. ${ }^{1}$ Usually after breast reconstruction is required a second surgical procedure to correct possible asymmetries in the contour of the breast that often does not meet the implant. Autologous fat grafting is a surgical technique that has been widely used for aesthetic refinement in post reconstruction breasts after mastectomies for diagnosis of breast cancer. You can also decrease the indication of more extensive and complex surgeries. In the treatment of breast cancer, surgery is performed, which can be partial or total resection of the breast, as one of the first choices. It is important to note the increased inclusion of Oncoplastic surgery in the world as a result of intent to improve the aesthetic result after a breast cancer operation, reducing the psychological impact on the patient, always acting under the security principle oncological. ${ }^{2}$ Although silicone breast implants are commonly used for breast augmentation, the adipose tissue has also been widely used in clinical routine for rehabilitation of these cases. ${ }^{3}$ Thus, the fat grafting is a technique that can improve or correct deformities caused by cancer, enabling the substitution of other conventional techniques complexidade ${ }^{4}$ or greater morbidity. Especially through the use of autologous fat grafting can still restore the contour, increase the volume and improve the irradiated skin in breast reconstruction surgery compromised by cancer especially after treatment. ${ }^{5}$ The purpose of this study is to prove the aesthetic effectiveness of autologous fat graft in post breast reconstruction refinement by presenting a case report. The methodology will be the literature review followed by a case report, which focuses on the practice an example of aesthetic rehabilitation with autologous fat grafting.

\section{Literature review}

Petit et al., ${ }^{6}$ conducted a retrospective study comprising three European institutions that treat cancer and breast reconstruction and concluded that fat grafting in breast reconstruction has a low complication rate and does not affect the radiological follow-up after conservative procedure breast but requires a study and control to prove the safety of cancer recurrence. Oliveira $\mathrm{Jr}$ et al., ${ }^{7}$ have sought to collect and identify, through systematic review the indications of fat grafting autologous breast and its safety through the reporting of clinical complications, radiological changes and incidence of breast cancer, primary or relapsed, the treated patients fat grafting with the topography of the breast. a systematic review of breast fat grafting was performed was carried out according to the guidelines of the PRISMA statement (Preferred Reporting Items for Systematic Reviews and Meta-Analyzes). They were included in this review 60 articles, totaling 4739 cases. The mammary fat grafting has been used satisfactorily for aesthetic and reconstructive treatment of the breasts. 155 were identified clinical complications, $60 \%$ of enduramento and/or palpable nodule. Its incidence, measured in 21 studies, It was $64 / 3015$. The authors concluded that there is a wide and effective clinical applicability for fat grafting autologous breast with a low complication rate and no evidence of impairment in the detection of breast cancer. With regard to cancer risk after surgical treatment of breast cancer, there are only three studies, totaling 636 cases accompanied and not identified significant differences in the cumulative incidence of breast cancer recurrence, though the small number of degree cases and low rating observed the design of these studies, the results were inconclusive, though seemingly safe. Bezerra et al., ${ }^{4}$ have sought to demonstrate a correction method breasts previously reconstructed contour defects by conventional techniques with fat graft after 8 years of experience. All patients included in this study were previously undergone mastectomy (total or partial) and some kind of breast reconstruction. The fat was obtained by liposuction method classic syringe, gently washed with saline solution in a stainless steel sieve, poured into a $60 \mathrm{cc}$ syringe after a few minutes and grafted. 112 patients were operated in the period 2005 to 2012. There was $1(8.9 \%)$ case of total loss of fat graft, $22(19.6 \%)$ patients had palpable nodules small, the average graft volume was $100 \mathrm{cc}$, and multiple procedures were required to attain the desired volume. 
Tsoi et al., ${ }^{8}$ compared the safety of the reconstruction of the tissue expander/implant reconstruction with autologous abdominal tissue. It performed a systematic review of the literature identified peerreviewed studies published from January 2000 to October 2012. The results suggest that the reconstruction of the expander/implant tissue have a higher risk of failure and reconstructive surgical site infection in compared to the reconstruction of autologous abdominal tissue. With the lack of long-term safety studies on different approaches to breast reconstruction, comparative studies of additional long-term are needed to support making evidence-based decisions. Coelho et al., performed a retrospective study cohort cross conducted by review of medical records of patients subjected to breast reconstruction with fat grafting. 18 patients were selected, and eight (44\%) had ductal tumor histology. Three patients (17\%) were submitted to neoadjuvant chemotherapy and of these, two (11\%) were also subjected to adjuvant chemotherapy; Nine $(50 \%)$ underwent only adjuvant chemotherapy. Eleven patients $(61 \%)$ underwent adjuvant radiotherapy, and thirteen (72\%) had hormonal treatment. As for breast reconstruction, eight patients $(44.4 \%)$ underwent TRAM six $(33.3 \%)$, more prosthesis and four expander $(22.2 \%)$, more large dorsal prosthesis. The number of fat grafting sessions ranged from one to four. It was not identified any cases of locoregional recurrence. At the conclusion of the authors, it was not observed any cases of locoregional recurrence, which adds, to date, reliability and security as fat grafting arsenal for procedures to improve the results of breast reconstruction after cancer treatment.

Piñeres et al., ${ }^{2}$ conducted a survey of the literature to show the mastopexy technique as superior pedicle oncoplastic surgical treatment for tumors T1, T2 and T3, located in the lower or central quadrant of the breast. The authors pointed out that the inclusion of oncoplásticas techniques in the treatment of breast cancer allows oncological results comparable to traditional surgery and satisfactory cosmetic results for the patient, avoiding disorders in her feminine identity. Further, to the authors, superior pedicle surgical technique is a Oncoplastic alternative with good results, which can be increasingly used in patients with tumors in the lower or central quadrants with large breasts or medium and ptosis, based on indications explained in detail in the text. Seyhan Tarhan et al., ${ }^{3}$ and described a new technique for breast augmentation using autologous dermal graft obtained from a simultaneous operation of abdominoplasty. The technique has been used on 8 patients of 4 breast requested abdominoplasty and mastopexy increase in the same session. First, the abdominal skin excess was deepithelialized. The dermal fat strips were collected in situ or extracorporeally this area. The prepared strips were then inserted into the pectoralis major muscle during mastopexy operation. Patients were followed for 7 months to 6 years. No infection or other technical related problems have been observed. Magnetic resonance imaging of a patient was obtained graft survival after 2 years. A slight increase, presumably from 50 to $100 \mathrm{ml}$, was noted by the breast in all patients.

Chia ${ }^{10}$ reported a case of aesthetic breast augmentation and discussed the review of the literature regarding the efficacy, the carcinogenic potential and imaging. The literature review was conducted in PubMed in English and in the Journal of Plastic Surgery, and the case report was given in the author's initial experience. As a result, it was observed that the volume remained stable from the second month, and there were no complications in the postoperative period. Imaging tests showed no pathological changes. They selected 24 related articles, and of these, only two prospective articles uncontrolled, but in general, there are no problems in diagnosis in imaging tests, no increased risk of carcinogenic potential, and the results are good in case series. It was concluded that the procedure is reproducible, safe and effective, consolidating itself as an indication in the restorative treatment of breast and an option to increase aesthetic. However, a longer learning curve may be required to prevent complications and achieve good results. Stumpf et al., 5 evaluated the local and systemic recurrence of breast cancer in patients undergoing autologous fat graft in the immediate reconstruction after conservative surgery for breast cancer. 167 patients were compared conserving surgery without undergoing reconstruction with 27 patients under conservative treatment with immediate reconstruction of the graft following Coleman's technique. All patients were suffering from carcinoma and were operated by a single surgeon between 2004 and 2011. The time of postoperative follow-up was 36 months. The results showed an overall incidence of local recurrence of $2.4 \%$. No patient's fat grafting group had local recurrence during the study period. For systemic recurrence rates were obtained 3, 7\% (one patient) for the fat grafting group and $1.8 \%$ (three patients) for the conserving surgery without reconstruction group. The authors concluded that there was no significant difference for local or systemic recurrence in the groups, wherein the graft immediately fat proved to be a safe procedure. Bezerra et al., ${ }^{4}$ said it essential to have science of recurrence impossibility before indicating the technique, the advantage we have operating time of less morbidity, liposuction bulkier anatomical region, obtaining body aesthetic result beyond the outcome of the breast does not require implants and as a possible disadvantage the percentage absorption may occur that it is impossible to measure, even if the technique is applied and there is little possibility of fat absorption. The surgical procedure is performed according to the sequence:

- starts with supine position, arms along the body;

- marking on the abdomen;

- infiltration with $1500 \mathrm{ml}$ saline with 2 ampoules of adrenaline wait 20 minutes;

- After manual liposuction cannula $3 \mathrm{~cm}$, the material centrifugation, decanting and transfer for $1 \mathrm{ml}$ syringes. Preparation $400 \mathrm{ml}$ of fat tissue;

- Incision site to receive the graft 40X12. Injection needle cannula fabric with $1.5 \mathrm{~cm}$ in tunnels back injection around the contour of the prosthesis $300 \mathrm{ml} 100 \mathrm{ml}$ right and left;

- Performing sutures and dressing.

\section{Clinical case report}

Female patient 32 years old, white, denies allergies, cancer, chronic diseases. Felt lump in her right breast seeking care with breast cancer specialist. On physical examination ptosadas breasts, flabby, no retractions. Directed thick biopsy needle 07/18/2014 with final award of invasive ductal carcinoma, Nuclear Grade 3 Grade 3 Histologic (Nottingham), and immunohistochemistry positive estrogen and progesterone receptor, Ki-67 positive $13 \%$. The patient was staged T3N1Mx-ECIIA being nominated three cycles of chemotherapy with cyclophosphamide, doxorubicin after being submitted to mastectomy and axillary dissection. During surgery mastectomy performed reconstruction with submuscular $275 \mathrm{cc}$ profile prosthesis round moderate and contralateral symmetrization with the same but with prosthesis implant Subglandular pexy by Peixoto technique. For aesthetic refinement effect in 2015 we opted for fat graft around the contour of the breast. 


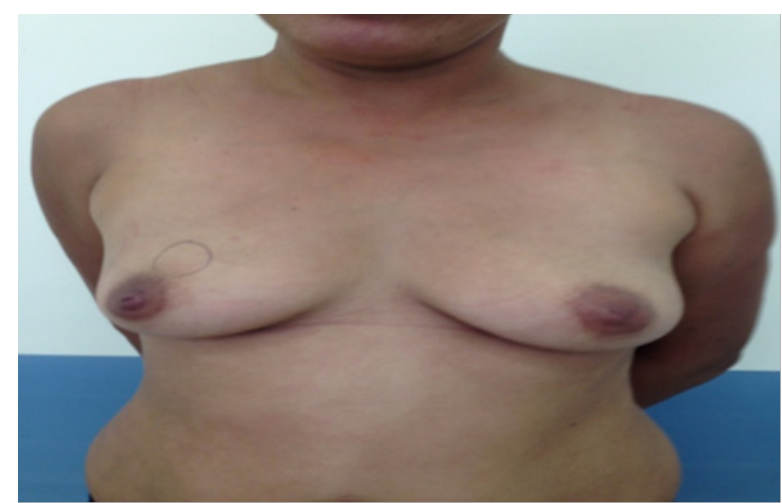

Figure I Premastectomy.

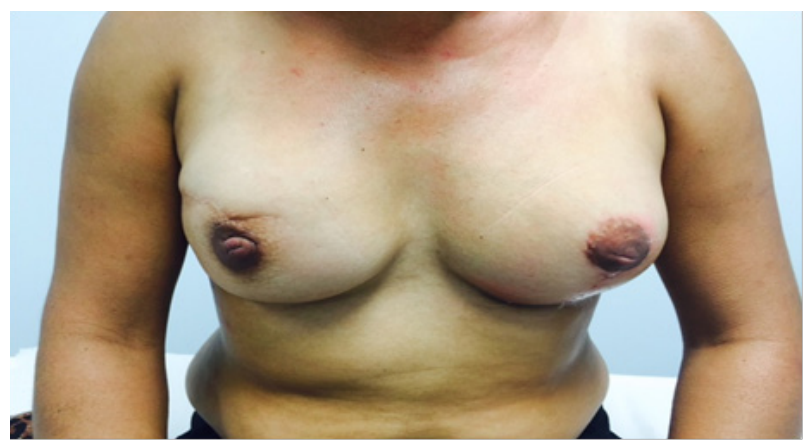

Figure 2 Post reconstruction.

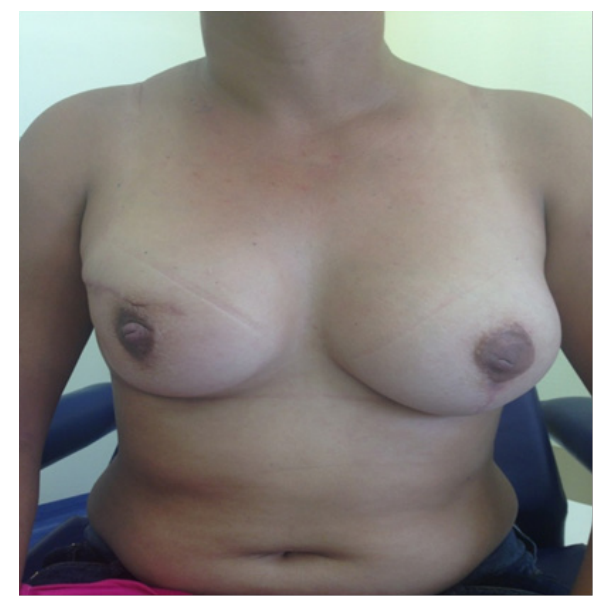

Figure 3 Reconstruction after 10 months.

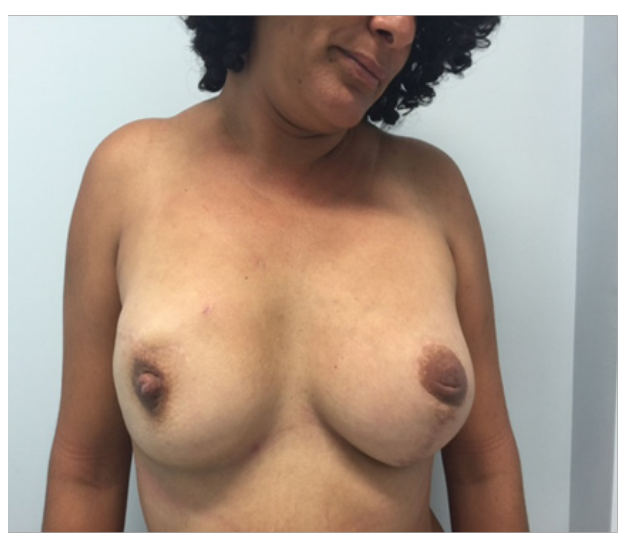

Figure 4 Post fat graft 60 days.

\section{Results and discussion}

The breast fat grafting can be a good solution to repair defects after processing and reconstruction of breast cancer and can also reduce more extensive surgery indication, such as the completion of flaps. Usually in these cases the postoperative complication rates are very low and there is little change in the follow-up mammograms. In our literature review, evident was the general consensus among different authors about the effectiveness of the application of the method and its advantages in promoting a better aesthetic standards and a better quality of life for women after mastectomy. The main advantages attributed to the method of autologous fat grafting were, according to Petit et al., ${ }^{6}$ and Oliveira Junior et al., ${ }^{7}$ the safety associated with the use of this method, since it is associated with a low complication rate and does not affect the radiological monitoring. Coelho et al., ${ }^{9}, \mathrm{Chia}^{10}$ and Stumpf et al., ${ }^{5}$ also agreed with these authors, highlighting in their review and reporting of clinical case, they were not found any cases of relapse. However, in terms of security, there was no unanimity among the authors, since, although it is considered a safe procedure, although there are doubts about the long-term effectiveness.

This was highlighted in the study by Tsoi et al., ${ }^{8}$ who said that there was a need for more comparative studies of additional long term to prove in practice. Also among the advantages, also for Bezerra et al., ${ }^{4}$ the method is assigned to a low morbidity and high satisfaction from both the professional and the patient. Nevertheless, the technique is not very easy for one to achieve the desired results, since many procedures should be performed until the satisfactory standard be glimpsed. However, it was also observed that, despite the good results obtained with the technique of employment, two main points still remain unclear: 1) how much fat is absorbed after grafting? 2) The potential risk of local tumor cells "dormant" are stimulated to induce a local recurrence? Specifically in the clinical case reported, the fat grafting technique has shown to be a good option for aesthetic refinement, this is because even after the reconstruction was possible to observe a breast asymmetry frame. Likewise, it is possible to observe that despite the breast prosthesis in the symmetrized be infraglandular were not observed with asymmetries large submuscular mastectomy breast prosthesis.

\section{Conclusion}

Based on literature reports and the clinical description of this case, it is concluded that the fat graft provides improved skin quality and aesthetics in general. Although the desired volume is not reached in a first session, satisfactory results may be obtained from the application of several sessions, adding excellent cosmetic results and improved quality of life for patients who have gone through this disease as devastating today.

\section{Acknowledgments}

None.

\section{Conflicts of interest}

None of the authors declares conflicts of interest.

\section{Funding}

None.

\section{References}

1. INCA. National Cancer Institute. Estimates of breast cancer in Brazil. 2018. 
2. Piñeres MDRS, BB Flores, CR Chagas, et al. Oncoplastic surgery technique of strapped to the superior pedicle. Rev Bras Mastologia. 2016;26(4):193-197.

3. Seyhan A, Tarhan S. Autologous breast augmentation using abdominal fat dermis strip grafts: a preliminary report. Aesthetic Plast Surg. 2016;40(2): 266-276.

4. Bezerra FJF, Moura RMG, Maia Neto JD. Fat grafting for breast reconstruction. Rev Bras Cir Plast. 2013;28(2):241-246.

5. CC Stumpf, Biazus JV, Zucatto FSAE, et al. Immediate reconstruction with autologous fat grafting: influence in breast cancerregional recurrence. Rev Col Bras Cir. 2017;44(2):179-186.

6. Petit JY, Lohsiriwat V, Clough KB, et al. The oncologic immediate surgical complications and outcome of lipofilling in Breast Cancer Patients: a multicenter study- Milan Paris-Lyon experience of 646 lipofilling procedures. Plast Reconstr Surg. 2011;128(2):341-346.
7. Oliveira Junior FC, Figueiredo JCA, Zampar AG, et al. Indications of autologous fat grafting breast. Rev Bra. Cir Plast. 2012;27(3 Suppl 1):56.

8. Tsoi B, NI Ziolkowski, Thomas A, et al. Safety of tissue expander/implant versus abdominal autologous breast tissue in breast reconstruction postmastectomy cancer patients: a systematic review and meta-analysis. Plast Reconstr Surg. 2014;133(2):234-249.

9. GVBF rabbit, Walnut FVM, Silva Junior VF, et al. Oncological evaluation after breast reconstruction with fat grafting. Rev Bras Cir Plast. 2014;29(2):243-247.

10. Chia CY. Breast augmentation with autologous fat grafting: literature review and case report. Rev Bras Cir Plast. 2016;31(1):105-111. 